Artículo

\title{
Caracterización de extensionistas rurales en México una contribución desde el análisis de correspondencias
}

\author{
Víctor Manuel Santos Chávez ${ }^{1 \S}$ \\ María de Jesús Santiago Cruz ${ }^{1}$ \\ Miguel Ángel Martínez Damián ${ }^{1}$ \\ Vinicio Horacio Santoyo Cortés ${ }^{2}$ \\ Adolfo Guadalupe Álvarez Macías ${ }^{3}$
}

${ }^{1}$ Colegio de Postgraduados-Campus Montecillo. Carretera México-Texcoco km 36.5, Montecillo, Texcoco, Estado de México. CP. 56230. (ecomjsc@colpos.mx; ange101@colpos.mx). ${ }^{2}$ Universidad Autónoma Chapingo-Centro de Investigaciones Económicas, Sociales y Tecnológicas de la Agroindustria y la Agricultura Mundial. Carretera México-Texcoco km 38.5, Chapingo, Texcoco, Estado de México. (hsantoyo@ciestaam.edu.mx). ${ }^{3}$ Universidad Autónoma Metropolitana-Xochimilco-Departamento de Producción Agrícola y Animal. Calzada del Hueso núm. 1100, Col. Villa Quietud, Coyoacán, Ciudad de México, México.

${ }^{\S}$ Autor para correspondencia: vsantoschavez@ gmail.com.

\section{Resumen}

La innovación rural sigue siendo un elemento crucial para resolver problemas de baja productividad en la agricultura y pobreza rural, la extensión rural constituye un instrumento de política agrícola que promueve la innovación a través de sus extensionistas. Con el propósito de caracterizar el perfil de estos actores, se realizó una tipología con base en sus atributos

profesionales y los problemas que enfrentan. Se empleó la encuesta a extensionistas del sistema de monitoreo y evaluación de la política de extensión rural 2016-2017, ésta recolectó información en diez estados de la república y su tamaño muestral fue de 609 cuestionarios. La metodología utilizó procedimientos estadísticos multivariados, en un primer momento el análisis de correspondencias estimó dimensiones principales, posteriormente se construyeron clústers para definir la tipología. Se detectaron tres estratos que agruparon problema predominante y área de la carrera profesional, ello permite una mejor comprensión de las características de los extensionistas y establecer estrategias de solución de problemas en la política de extensión centrada en el conocimiento de las capacidades y condiciones de uno de sus principales actores.

Palabras clave: desarrollo rural, educación rural, formación agrícola, tipologías rurales.

Recibido: agosto de 2021

Aceptado: octubre de 2021 


\section{Introducción}

A nivel internacional existe amplio consenso sobre la importancia de la innovación rural como factor decisivo para resolver problemas estructurales en la agricultura, tales como bajos ingresos de los productores y baja productividad de la actividad (Ardila, 2010; Christopolos, 2010). El acceso de los agricultores a tecnologías agrícolas y prácticas de intercambio de conocimientos es reconocido como un elemento fundamental en la solución de dichos problemas. En este sentido, el extensionismio rural es una arista fundamental en los procesos de innovación, alude a una función educativa no formal y a mecanismos de transferencia de conocimiento a los agricultores y a otros actores que conforman los sistemas agroalimentarios dentro de una estructura socioeconómica rural.

Esta labor es especialmente relevante en los sistemas productivos más vulnerables, donde es necesario explorar nuevas oportunidades para mejorar la seguridad alimentaria, acceder al sistema financiero y comercial, mitigar las vulnerabilidades ambientales y lograr representatividad en el ámbito político y social.

En estos procesos de transferencia de tecnologías y conocimiento, los productores y los extensionistas rurales tienen un papel preponderante. Los extensionistas son un actor clave para promover la innovación rural en los predios; juegan un rol prominente en la adopción de tecnologías y conocimiento entre los productores rurales, adicionalmente pueden alentar la organización de productores y el acceso a mercados, entre otros aspectos, lo que representa un coadyuvante clave en la construcción de alternativas de solución a los problemas de los productores agropecuarios.

En consecuencia, el análisis de las políticas de extensión rural ha cobrado nuevamente relevancia recientemente; sin embargo, a la par se advierte que a pesar de la importante labor de los extensionistas hay un descuido en el análisis de su desempeño, así como de los problemas que encuentran en el desarrollo de sus actividades (Cook et al., 2021). En esta investigación se parte del supuesto de que el extensionista es un actor fundamental en los procesos de innovación rural; a partir, de su labor de articulación entre diferentes actores: productores, comercializadores, industria, centros de investigación y de transferencia de tecnología e innovación. Por tanto, se asume que analizar los problemas que los extensionistas perciben en sus rutinas de trabajo es una herramienta valiosa para identificar, analizar y proponer alternativas para elevar la eficiencia de su trabajo y en última instancia, la de las políticas de extensión rural.

En este marco, el objetivo de la investigación fue describir y analizar el perfil de los extensionistas rurales en México y articular dicho análisis con los problemas que han observado en el desarrollo de su actividad profesional. La primera parte del documento contiene el procedimiento metodológico de la investigación, se precisan las bases de datos utilizadas y los procedimientos estadísticos empleados. La segunda incluye los resultados y su discusión, centrada en la descripción del perfil de los extensionistas, el análisis de sus problemas y un enfoque que agrupó problemas principales con la variable del perfil profesional. El último apartado contiene las conclusiones. 


\section{Materiales y métodos}

En 2016 y 2017 la Organización de las Naciones Unidas para la Alimentación y la Agricultura (FAO) evaluó el Componente de Extensionismo que implementó la entonces denominada Secretaría de Agricultura Ganadería, Desarrollo Rural, Pesca y Alimentación (SAGARPA), hoy Secretaría de Agricultura y Desarrollo Rural. Con 609 cuestionarios aplicados a igual número de extensionistas en los dos años considerados se integró una base de datos que sustenta la investigación cuantitativa, principalmente descriptiva. La base de datos incluyó información de extensionistas en diez estados de la república. En cada estado se aplicó un muestreo aleatorio simple con representatividad estatal, con un nivel de confianza de $90 \%$ y un error de $10 \%$.

La selección de estados no obedeció a criterios probabilísticos, la encuesta se realizó en los estados donde se establecieron procesos de monitoreo y evaluación de la política de extensión rural, lo cual imposibilita una inferencia a nivel nacional. Sin embargo, considerando que durante el periodo 2016-2017 se contrataron 7114 extensionistas a nivel nacional (SAGARPA y FAO, 2018; SAGARPA y FAO, 2018b), el tamaño muestral equivale 8.6\% del total de extensionistas en México. El (Cuadro 1) presenta las características de la muestra, dividida por estado, tamaño de muestra, sexo, edad, años de experiencia y área de formación profesional de los extensionistas.

Cuadro 1. Características generales de la muestra.

\begin{tabular}{|c|c|c|c|c|c|c|c|c|c|c|}
\hline \multirow{2}{*}{ Estado } & \multirow{2}{*}{$\mathrm{n}$} & \multicolumn{2}{|c|}{$\operatorname{Sexo}(\%)$} & \multirow{2}{*}{ Edad } & \multirow{2}{*}{$\begin{array}{c}\text { Años de } \\
\text { experiencia }\end{array}$} & \multicolumn{5}{|c|}{ Formación académica (\%) } \\
\hline & & $\mathrm{H}$ & $\mathrm{M}$ & & & Agronomía & Zootecnia & Sociales & Biología & Otras \\
\hline Chiapas & 82 & 79.3 & 20.7 & 36.9 & 2 & 46.3 & 37.8 & 2.4 & 1.2 & 12.2 \\
\hline Durango & 60 & 81.7 & 18.3 & 45.7 & 2.4 & 43.3 & 41.7 & 10 & 0 & 5 \\
\hline Edo. México & 73 & 65.8 & 34.2 & 39.2 & 2.8 & 46.6 & 41.1 & 6.8 & 0 & 5.5 \\
\hline Guanajuato & 72 & 70.8 & 29.2 & 43.7 & 4.1 & 31.9 & 56.9 & 8.3 & 2.8 & 0 \\
\hline Michoacán & 62 & 59.7 & 40.3 & 42 & 4.3 & 37.1 & 41.9 & 14.5 & 4.8 & 1.6 \\
\hline Oaxaca & 85 & 75.3 & 24.7 & 37.4 & 2 & 61.2 & 16.5 & 7.1 & 10.6 & 4.7 \\
\hline Puebla & 27 & 74.1 & 25.9 & 40.3 & 2.4 & 37 & 40.7 & 7.4 & 7.4 & 7.4 \\
\hline San Luis Potosí & 41 & 87.8 & 12.2 & 48.2 & 2 & 36.6 & 56.1 & 4.9 & 0 & 2.4 \\
\hline Sinaloa & 70 & 82.9 & 17.1 & 42.8 & 3 & 44.3 & 27.1 & 15.7 & 7.1 & 5.7 \\
\hline Sonora & 37 & 75.7 & 24.3 & 46.4 & 3.2 & 35.1 & 18.9 & 24.3 & 2.7 & 18.9 \\
\hline Total & 609 & 74.9 & 25.1 & 41.6 & 2.8 & 43.5 & 37.3 & 9.5 & 3.8 & 5.9 \\
\hline
\end{tabular}

\section{Análisis estadístico}

Se utilizó la metodología de análisis de correspondencia (AC) para estudiar relaciones entre variables categóricas. El AC es una extensión adaptada de la metodología de análisis de componentes principales, se utiliza para el manejo estadístico de datos categóricos obtenidos en encuestas sociales. El AC identifica dimensiones para las cuales se advierte poca dispersión en perfiles o grupos y elimina las direcciones de dispersión que aportan poca información, reduciendo con ello la dimensionalidad (Greenacre, 2007). A partir del análisis de tablas de contingencia con datos de frecuencias numéricas permite una representación gráfica y simple que proporciona la comprensión de un gran número de datos (Greenacre, 2007; Abdi y Williams, 2010). 
El principio del AC es generalizar una representación gráfica de un diagrama de dispersión, donde se representan datos en forma de puntos con relación a los ejes de coordenadas perpendiculares: el eje horizontal (eje de las x) y el eje vertical (eje de las y). El AC permite analizar el comportamiento correlacional entre variables categóricas en una o varias dimensiones, además, de estas dimensiones se pueden establecer hipótesis de interpretación de la población incluida en la muestra.

El AC se ha utilizado en estudios sociales para definir tipologías de productores rurales (Bouyer $e t$ al., 2011; Urcola et al., 2015; Fantappiè et al., 2020). En la presente investigación el AC fue utilizado para analizar el perfil agregado de los extensionistas en México, principalmente la relación entre el tipo de carrera estudiada y las dificultades que presentan estos actores en el desempeño de sus actividades en los programas de extensión rural. Se utilizó una prueba de jicuadrada para evaluar el nivel de significancia de los resultados obtenidos. El software utilizado para el análisis estadístico fue RStudio.

\section{Resultados y discusión}

Del total de profesionistas consultados, $74.9 \%$ fueron hombres y el resto mujeres. Al contrastar estos resultados con otros similares en América Latina, se advierte que la baja prevalencia de mujeres profesionistas en las políticas de extensión es una condición recurrente en varios países de la región. En un estudio en 10 países de América Latina, se halló que en promedio la población masculina representa $70 \%$ del total de extensionistas (Landini, 2016). Este escenario contrasta con las nuevas tendencias en el diseño de políticas de extensión más plurales que buscan fortalecer la capacidad institucional de las organizaciones dentro de la política de extensión con la incorporación del enfoque de género.

A partir del reconocimiento de que tradicionalmente los servicios de extensión se orientan hacia agricultores varones, a través de extensionistas varones en su mayoría, autores como Qamar (2011) señalan que existe evidencia internacional que muestra la efectividad en el impacto de las políticas de extensión cuando la estrategia incluye dos ámbitos: servicios de extensión orientados exclusivamente a mujeres y que sean brindados a través de mujeres extensionistas.

Si bien hay experiencias en países de los continentes africano (Lahai et al., 1999) y asiático (Lamontagne-Godwin et al., 2017) donde, con base en análisis estadísticos, se han examinado los resultados de los servicios de extensión brindados por hombres y mujeres extensionistas, y no se han encontrado diferencias estadísticamente significativas, el debate internacional ha ponderado que el enfoque de género en la extensión rural puede representar una estrategia valiosa por el papel que las mujeres desempeñan en la agricultura y en los sistemas agroalimentarios, sobre todo en países de América Latina.

Al analizar la edad de los extensionistas se encontró un promedio de 41.6 años, al desagregar el análisis por sexo, se advierte que las mujeres reportaron una edad media menor, de 36.3 años, mientras que para los hombres fue de 43.4 años. Esto permite inferir que se trata de una población relativamente joven, al estratificar el análisis por grupo de edad, resulta relevante observar que $22 \%$ de los extensionistas son personas con una edad menor a los 30 años, en el extremo opuesto se ubicó el estrato de entre 50 y menos de 60 años (21.3\%). El grupo más importante en términos relativos fue el de 30 a menos de 40 años, que representa casi una tercera parte del total de extensionistas. 
El $82.3 \%$ de extensionistas tiene licenciatura terminada, lo que es congruente con el diseño de la política de extensión en el periodo estudiado, cuya normatividad establecía la contratación de profesionistas con al menos una carrera terminada en el área de las ciencias naturales. En este sentido sobresalen dos ámbitos, el primero es que $2.1 \%$ de actores contratados reportó contar con carrera técnica terminada, y en el extremo opuesto $15.6 \%$ declaró contar con un posgrado. Asimismo, $0.7 \%$ de los extensionistas en México tiene grado de doctor en ciencias y $14.9 \%$ cuenta con maestría en ciencias.

Del total de profesionistas contratados para llevar a cabo procesos de extensionismo, $43.5 \%$ tiene carrera relacionada con la agricultura/agronomía y $37.3 \%$ carreras del área de ganadería/zootecnia. Los profesionales con carrera afín a ciencias sociales representan 9.5\% del total. La mayoría de los extensionistas son agrónomos generales y especialistas en fitotecnia y zootecnia, en conjunto éstos representan $36.6 \%$ del total por otra parte, los médicos veterinarios zootecnistas son una población importante en términos relativos, constituyen $26.3 \%$ del total de extensionistas. El 3.8\% de extensionistas tiene carreras ajenas al desarrollo rural, tanto en términos técnicos como sociales.

Se encontraron profesionales con carreras en derecho, química, físico-matemático, informática/computación, psicología y relaciones internacionales. El perfil profesional muestra que la extensión rural en México constituye un proceso que ha considerado marginalmente los múltiples factores sociales y políticos que dan forma a la vida rural en cambio, se ha privilegiado el acceso a procesos de adopción de tecnologías mediados por prácticas fundamentalmente técnicas.

El acceso de los productores a tecnologías agrícolas es reconocido por la política de extensión como el elemento crucial de sus acciones, que asume que es un factor fundamental para mejorar sus medios de vida. Sin embargo, se ignora que las prácticas de extensión se basan en procesos socioeconómicos, políticos y culturales que caracterizan a los medios de vida rural, esto muestra cierta desconexión del servicio de extensión con las realidades complejas de los agricultores, así como de sus relaciones y prácticas. Cook et al. (2021) argumentan sobre la importancia de introducir la extensión al mundo real a través de transferir conocimientos y tecnologías a los agricultores no solo desde un ámbito productivo, sino desde diferentes enfoques que construyan conocimiento complejo capaz de dar cuenta y solución de los múltiples problemas que enfrentan los actores del medio rural.

En el caso mexicano, se advierte poca participación de profesionistas de áreas sociales, económicas y políticas. La ausencia de ciertos perfiles técnicos revela la falta de atención a temas estratégicos que tienen relación en la solución de problemas como: la conservación de los recursos naturales, la organización social de productores y temas relativos a mercado y análisis de riesgos, entre otros.

\section{Análisis de problemas de los extensionistas}

El Cuadro 2 sintetiza los problemas que los extensionistas definieron con base en información sobre el desempeño crítico de su labor e indica el incumplimiento de metas o consecuencias inesperadas dentro de la política 2016-2017 de extensionismo rural en México. 
Cuadro 2. Principales problemas de los extensionistas en México.

\begin{tabular}{|c|c|c|c|c|c|c|}
\hline \multirow[b]{2}{*}{ Problema } & \multicolumn{5}{|c|}{ Formación académica } & \multirow[b]{2}{*}{$\overline{\mathrm{x}}(\%)$} \\
\hline & $\begin{array}{l}\text { Agric./ } \\
\text { agron. }\end{array}$ & $\begin{array}{c}\text { Ganad./ } \\
\text { zootecnia }\end{array}$ & $\begin{array}{l}\text { Soc./ } \\
\text { económ. }\end{array}$ & Biología & Otras & \\
\hline $\begin{array}{l}\text { Falta de oportunidad respecto a calendarios } \\
\text { productivos }\end{array}$ & 65.8 & 19.8 & 7.2 & 1.8 & 5.4 & 19 \\
\hline Falta de continuidad a los grupos de extensión & 41.7 & 43.8 & 8.3 & 1 & 5.2 & 16.4 \\
\hline Capacitación a extensionistas productivista & 27.1 & 49.4 & 10.6 & 5.9 & 7.1 & 14.5 \\
\hline Precariedad laboral de extensionistas & 40.3 & 33.9 & 16.1 & 4.8 & 4.8 & 10.6 \\
\hline $\begin{array}{c}\text { Falta de articulación de la política de } \\
\text { extensión }\end{array}$ & 37.5 & 37.5 & 10.4 & 4.2 & 10.4 & 8.2 \\
\hline $\begin{array}{l}\text { Servicios de corta duración con grupos de } \\
\text { beneficiarios }\end{array}$ & 39.4 & 42.4 & 9.1 & 3 & 6.1 & 5.6 \\
\hline Baja vinculación entre grupos de beneficiarios & 60.6 & 24.2 & 12.1 & 0 & 3 & 5.6 \\
\hline $\begin{array}{l}\text { Excesivos procesos burocráticos durante el } \\
\text { servicio }\end{array}$ & 45.2 & 41.9 & 3.2 & 6.5 & 3.2 & 5.3 \\
\hline $\begin{array}{c}\text { Escasa coordinación al interior del } \\
\text { Componente }\end{array}$ & 38.1 & 33.3 & 14.3 & 4.8 & 9.5 & 3.6 \\
\hline $\begin{array}{c}\text { Burocratización de procesos de monitoreo y } \\
\text { evaluación }\end{array}$ & 25 & 50 & 0 & 15.0 & 10 & 3.4 \\
\hline $\begin{array}{c}\text { Selección de extensionistas sin criterios } \\
\text { objetivos }\end{array}$ & 62.5 & 37.5 & 0 & 0 & 0 & 2.7 \\
\hline $\begin{array}{c}\text { Servicios al margen de demandas de } \\
\text { beneficiarios }\end{array}$ & 42.9 & 42.9 & 7.1 & 7.1 & 0 & 2.4 \\
\hline $\begin{array}{c}\text { Excesivas reuniones para gestión del } \\
\text { Componente }\end{array}$ & 11.1 & 66.7 & 22.2 & 0 & 0 & 1.5 \\
\hline Capacitación a beneficiarios productivista & 0 & 50 & 16.7 & 16.7 & 16.7 & 1 \\
\hline
\end{tabular}

Basado en BD (2016-2017).

La definición de problemas se realizó con base en la frecuencia en que cada uno fue señalado por los extensionistas. El principal problema fue la falta de oportunidad respecto a calendarios productivos (1). En este sentido, 19\% de extensionistas identificó como principal preocupación la baja correspondencia entre los inicios de los servicios de extensión y los ciclos productivos en los predios de los productores. Advierten que esta situación tiene un efecto en la toma de decisiones a nivel de predio, pues cuando ellos inician sus funciones en las unidades productivas, las decisiones técnicas más importantes ya fueron tomadas por los productores.

La evaluación nacional de la política de extensión en 2017 concluyó que 94\% de extensionistas inició actividades entre junio y agosto, lo que técnicamente muestra que llegan al predio cuando los productores ya han tomado las decisiones de qué producir y cómo hacerlo, al menos para los procesos esenciales como la siembra, principalmente en agricultura de temporal o los periodos de pariciones en ganadería (SAGARPA y FAO, 2018b). Se dispone de evidencia empírica que muestra una correlación positiva entre la oportunidad en el inicio de los servicios y el incremento de la productividad (Santoyo-Cortés et al., 2016). 
En segundo lugar, $16.4 \%$ de entrevistados observó el problema relativo a la falta de continuidad de los grupos de extensión (2); conceptualmente las políticas de extensión rural constituyen una estrategia para el logro de metas, para la efectividad y la legitimidad de éstas juega un papel importante contar con procesos duraderos donde los grupos de extensión y productores tengan continuidad a mediano plazo. En tercer lugar, se señaló la capacitación a extensionistas productivista (3). La orientación de la política de extensión muestra que la estrategia se ha centrado en un ámbito preponderantemente productivo.

El discurso que define la relación entre agricultores y extensionistas enfatiza que la solución de problemas se logrará con un enfoque productivista por ello, las capacitaciones que los extensionistas reciben de parte de la administración pública se basan en un conjunto de prácticas tecnológicas de corte técnico y productivo. Sin embargo, los extensionistas han encontrado en la praxis que este enfoque no solo ignora los procesos socio-culturales, económicos y políticos que dan forma a los medios de vida rural, sino que esto deriva en una política de extensión desconectada de las realidades de los agricultores.

Las prácticas de capacitación a extensionistas centradas en el ámbito productivo constituyen mecanismos excluyentes que establecen límites que legitiman un enfoque donde no se parte del principio de reconocer los problemas del medio rural. Autores como Bartlett (2010) advierten que estos procesos tienen como consecuencia que los extensionistas ignoren los procesos sociopolíticos, económicos y culturales que dan forma a la vida rural. Estos procesos se relacionan a su vez con otro problema señalado por los extensionistas y es el de la capacitación a beneficiarios por parte de los extensionistas demasiado productivista (14).

El problema precariedad laboral de extensionistas (4) es un tema de la capacidad institucional existente para implementar la política de extensionismo rural. En este sentido los extensionistas identifican que no hay procesos eficientes en el pago de sus honorarios, remarcan además falta de esquemas de seguridad social y nulos apoyos para gastos de operación. Esta situación ha sido reportada en diversos países en América Latina, donde hay una contratación de extensionistas generalmente temporal y con una alta rotación del personal, lo que repercute en la calidad de los servicios de extensión (Ardila, 2010).

En quinto lugar, se observó la falta de articulación de la política de extensión (5) relativo a que los paquetes tecnológicos que se difunden no consideran las limitaciones productivas de la población objetivo. Los entrevistados plantean la necesidad de articular servicios de extensión con inversiones auxiliares para apoyar los cambios tecnológicos impulsados. El 5.6\% de extensionistas identificó como problema los servicios de corta duración a grupos de beneficiarios (6). Análisis de SAGARPA y FAO $(2018,2018 b)$ muestran que durante los años 2017 y 2018 la duración promedio de los servicios fue de 7.1 y 8.1 meses respectivamente, esto da cuenta de servicios de corta duración, lo que en la narrativa de los extensionistas imposibilita desarrollar plenamente sus servicios y cubrir al menos un ciclo productivo completo.

A nivel internacional los efectos de este obstáculo han sido analizados y hay una convicción de que políticas de extensión de corto plazo son ineficientes en el logro de objetivos de productividad y transferencia de conocimientos (Solleiro et al., 2020). Además, se ha observado que los procesos de extensión rural no han fortalecido la organización a través de la vinculación entre grupos de beneficiarios, por ello se advierte como problemática la baja vinculación entre grupos de beneficiarios (7), en este sentido los extensionistas encuentran imprescindible desarrollar estrategias donde haya mayor vinculación entre grupos de beneficiarios. 
Los problemas de excesivos procesos burocráticos durante el servicio (8) y durante los procesos de monitoreo y evaluación (10) son dos dimensiones, a decir de los extensionistas, que hacen referencia a dificultades en la habilidad del aparato institucional para desempeñar las tareas con efectividad y eficiencia. A estos problemas se suma la escasa coordinación al interior de las dependencias en el staff que ejercen la coordinación de la operación de la política de extensión (9).

Estudios previos han documentado la gama de instancias imbuidas en la operación de la política de extensión en México (Santos et al., 2019). La continuidad de este problema en los años 2016 y 2017 muestra una ausencia en el cambio de rutinas de operación de las llamadas dependencias staff que ha generado cuellos de botellas en los procesos burocráticos de la política, precisamente como el problema de excesivas reuniones del componente (13).

El 2.7\% de entrevistados señaló como problemática la selección de extensionistas sin criterios técnicos (11). Tal como se señaló en este estudio, los hallazgos muestran la contratación de profesionales del área del derecho, la química, las relaciones internaciones, la física y la matemática, lo que desde la perspectiva de la mayoría de extensionistas no coadyuva en la resolución de problemas productivos de los predios, sobre todo cuando se trata de programas de trabajo unipersonales. Otro elemento crítico muestra que los servicios de extensión se encuentran al margen de las demandas de los beneficiarios (12), una hipótesis deriva de que la labor del extensionista se centra en atender lo que marca las rutinas burocráticas de la política de extensión en lugar de las demandas específicas del productor.

\section{Correspondencia entre problema identificado y perfil profesional de extensionistas}

En el Cuadro 3 se observan la representación de los cuatro vértices que constituyen los problemas de los extensionistas según su área profesional y los porcentajes de varianza explicada por cada dimensión. Al sumar los valores se puede conocer la varianza explicada por las dimensiones. Las dimensiones 1 y 2 explican 64.6 y $17.4 \%$ respectivamente del total, lo que corresponde a un porcentaje acumulado del total de varianza retenida por las dos dimensiones de $82.1 \%$.

Con base en la varianza explicada por cada eje como dimensión se puede expresar la suma de las dos dimensiones principales cuyo valor agregado es de 0.1169 , por ende, el estadístico ji-cuadrado de independencia entre dos variables es igual a 83.36975. Al aplicar una prueba estadística utilizando una distribución ji-cuadrada el $p$-value de la estimación arroja un valor altamente significativo $(p=0.003738235)$.

Cuadro 3. Descomposición de inercia por dimensión principal.

\begin{tabular}{cccc}
\hline Dim. & eigenvalue & $(\%)$ varianza & $(\%)$ varianza acumulada \\
\hline 1 & 0.09211995 & 64.64 & 64.64 \\
2 & 0.0248523 & 17.44 & 82.08 \\
3 & 0.01866507 & 13.1 & 95.18 \\
4 & 0.00687508 & 4.82 & 100 \\
\hline
\end{tabular}


La Figura 1 muestra la representación del mapa simétrico de la relación entre variables, lo que permite ver el grado de asociación entre carreras de extensionistas y categorías de problemas señalados. Ello facilita la interpretación de posiciones relativas y da una idea del comportamiento de variables. Los perfiles de agronomía/agricultura están relacionados con los problemas 1, 11 y 7 , mientras que las carreras del área de biología están más próximas a los problemas 10 y 14.

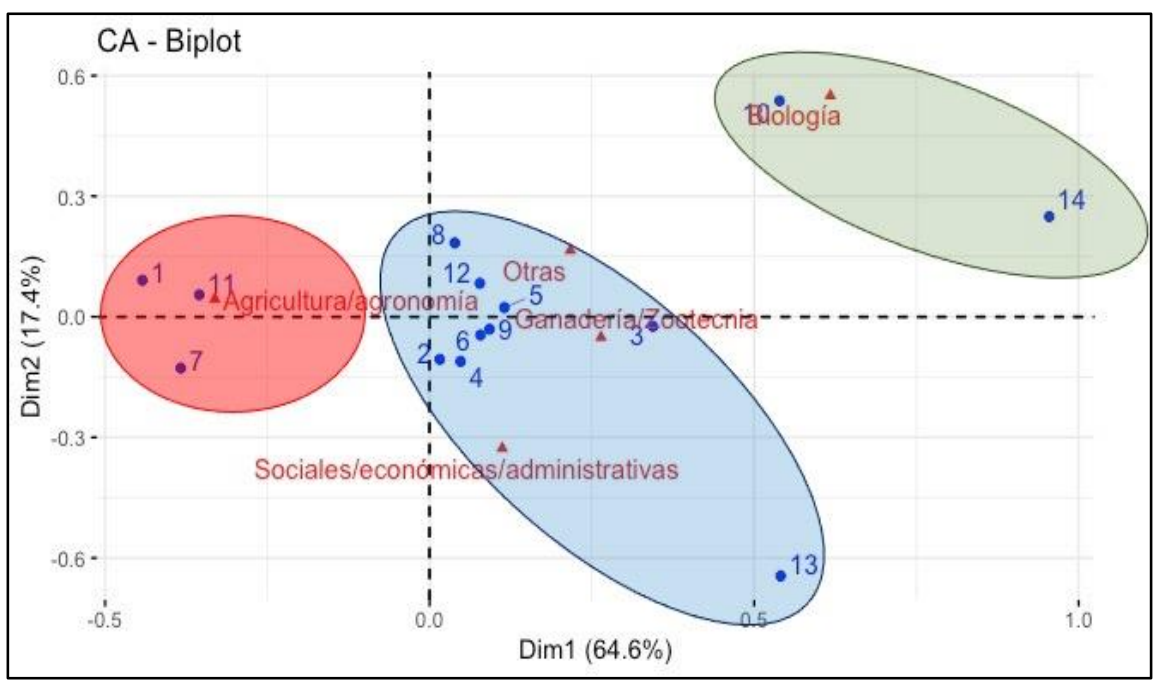

Figura 1. Mapa simétrico de relación entre datos.

El mapa permite identificar el patrón global dentro de los datos, las filas están representadas por puntos azules y las columnas por triángulos rojos. La distancia entre cualquier fila de puntos o columna da una medida de su similaridad (disimilaridad). Tres grupos están claramente identificados a partir del AC. Al incluir un análisis factorial con base en un dendograma de clúster, se comprobó la delimitación de tres grupos (Figura 2). Los extensionistas fueron discriminados en tres estratos.

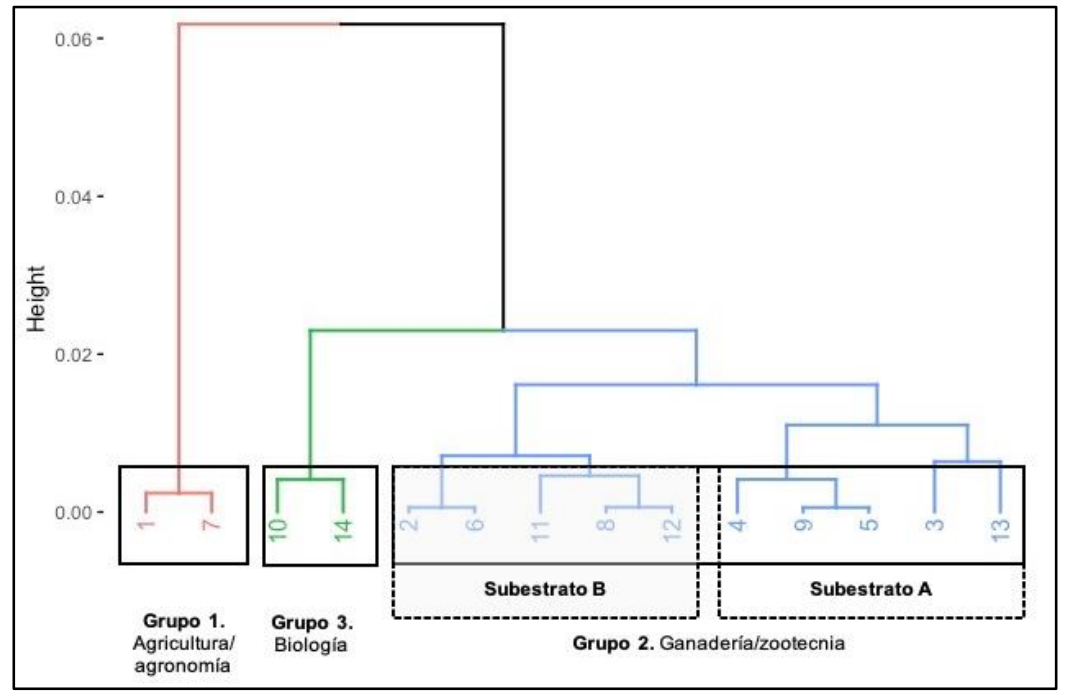

Figura 2. Dendograma del análisis de clúster. 
El primer grupo incluyó a $43.5 \%$ de ellos, corresponde con profesionales de las carreras de agricultura y agronomía, a este grupo se asociaron los problemas 1 y 7 , el segundo abarcó profesionistas del área de ganadería/zootecnia, este grupo tiene un listado mayor de problemas que los agrupa en un estrato (problemas 2, 6, 11, 8, 12, 4, 9, 5, 3, 13), el último grupo incluyó a extensionistas con carrera en biología, a quienes agruparon los problemas 10 y 14 .

\section{Descripción y análisis de cada clúster identificado}

El grupo 1. (agricultura/agronomía) estuvo asociado a problemas de falta de oportunidad de los servicios de extensión respecto a calendarios productivos y baja vinculación entre grupos de beneficiarios. Es el grupo más amplio de extensionistas, los cuales registran una edad media de 42.5 años, con base en su experiencia en las políticas recientes de extensión ( 2.7 años) advierten que las estrategias de adopción de tecnologías centradas en el incremento de la producción requieren oportunidad en el inicio de servicios, y que los promotores lleguen a la parcela con antelación a que los productores instrumenten sus actividades productivas.

Esta articulación entre la toma de decisiones de productores y estrategias de extensión cobra mayor relevancia en servicios orientados al ámbito agrícola, donde el establecimiento oportuno de la estrategia de extensión incluye procesos de difusión y adopción de tecnologías muy amplios, que van desde la selección de cultivo y variedades, tecnologías que abarcan maquinaria, equipo, así como programas de fertilización y aplicaciones de otros agroquímicos, y buenas prácticas en el manejo de labores culturales, incluso la forma en que los productores se insertan en las cadenas de compra de insumos (Aguilar-Gallegos et al., 2015).

Dado que fue un tema que principalmente destacaron los profesionales dedicados a las actividades agrícolas, es posible que este problema sea más marcado en este subsector que en el pecuario. El problema de baja vinculación entre grupos da cuenta de que estos actores opinan que las estrategias de innovación orientadas a mejorar el ámbito productivo, por sí mismas son insuficientes, dado que se requieren procesos de interacción con mayor frecuencia entre productores y extensionistas.

El grupo 2. (ganadería/zootecnia) representa 37.3\% del total de extensionistas, con una edad promedio de 41.3 años, constituye un grupo con alta heterogeneidad en relación con los problemas que el análisis estadístico asoció a su perfil. Derivado del dendograma del análisis de clúster se puede constatar que al interior de este grupo se identifican dos subestratos, uno interesado por cambiar el paradigma burocrático de las dependencias de la política de extensión y otro centrado en mejorar los servicios de extensión como papel de facilitador en los procesos de innovación.

En el primer subestrato A y el análisis agregado muestra la preocupación por mejorar el desempeño de las dependencias gubernamentales de la política de extensión en México y establecer acciones orientadas a mejorar su organización, servicio y control, con el propósito de hacer más eficiente su proceso de gestión. Se identifican problemas orientados a establecer acciones para que las dependencias cumplan con las normas establecidas y mejorar la calidad de los servicios. Las objeciones de este grupo están relacionadas con la precariedad laboral de extensionistas, la escasa coordinación al interior de la administración pública, la falta de articulación de la estrategia de extensión con otras estrategias similares dentro de la política sectorial, así como excesivas reuniones a las que las rutinas burocráticas los constriñe asistir, además se suma el hecho de que las reuniones de capacitación mantienen un enfoque productivista. 
Este subgrupo establece una fuerte crítica a las dependencias encargadas de la implantación de la política de extensión, al juzgarlas a menudo como incapaces de cumplir y hacer cumplir las normas vigentes. El subestrato B mostró preocupaciones relativas a la continuidad de grupos, servicios de corta duración y que no responden a las necesidades de los productores, selección de extensionistas sin criterios técnicos y, al igual que en el agregado del grupo 2, no soslayaron problemas relativos a los excesivos procesos burocráticos de la política de extensión. Resulta claro para este subestrato que la promoción de tecnologías con base en políticas de corto plazo no generará resultados de productividad y transferencia de conocimiento en los productores, además, considerando que los servicios duran entre siete y ocho meses, las observaciones de este subestrato se orientan a desarrollar un vínculo más duradero entre extensionistas y productores.

Con base en estos problemas se advierte un sesgo de la política de extensión, es poco probable lograr resultados con estrategias discontinuas y servicios de corta duración. La teoría clásica de la difusión de innovaciones da cuenta de la importancia en establecer interacciones duraderas y relevantes para persuadir al menos a una minoría de productores (pioneros y adaptadores) en aplicar las innovaciones propuestas (Rogers, 2003). Esto nuevamente muestra la centralidad que los extensionistas conceden a los procesos de interacción social, lo que concuerda con diversos estudios que muestran que dado que convencionalmente las intervenciones y metodologías de los programas de extensión rural incluyen procesos de acompañamiento de productor a productor, donde se requiere la identificación de productores pioneros que hayan adoptado innovaciones para que funjan como actores clave en la difusión de tecnologías entre sus pares (Cook et al., 2021), lo que difícilmente se transformará en resultados trascendentales si se mantienen intervenciones de corto plazo y servicios con duración menor a un año.

Además, en este subgrupo se advierte la preocupación por la ineficiencia de los servicios de extensión, dado que éstos no suelen responder a los problemas de los beneficiarios, lo que en parte lo atribuyen a la falta de procesos técnicos en la contratación de extensionistas, pues a su parecer hay perfiles de profesionales que no concuerdan con las estrategias sectoriales de la política de extensión. El marco institucional de las políticas de extensión ha descuidado el desarrollo de capacidades en los extensionistas, así como el ajuste de su perfil profesional para que puedan responder a los nuevos retos de la agricultura mundial.

El grupo 3. (biología) mostró una preocupación por la prevalencia de un modelo de extensión productivista, orientado a la producción primaria, que descuida la complejidad de la agricultura y la emergencia de nuevos retos en la producción. Es interesante observar que este grupo da cuenta de una minoría de extensionistas preocupados por la emergencia de nuevas tendencias en la agricultura. Lo que concuerda con el análisis de organismos internacionales como la FAO que han visibilizado la importancia de ampliar las funciones de la extensión rural y hacerla más plural e incluir cuestiones relativas a mercados, organización, servicios financieros, cooperativismo, agroindustria, gestión de recursos naturales, desarrollo de actividades rurales no agropecuarias, entre otras (Christoplos, 2010).

En la actualidad se advierte que los problemas de la agricultura se han complejizado. A pesar de que sigue vigente la necesidad de promover innovaciones agrícolas que mejoren la productividad, la rentabilidad y la sustentabilidad de los agricultores, existe un creciente reconocimiento que pone en duda la efectividad de los modelos de extensión que se basan en la predominancia de perfiles de sus extensionistas de corte productivista (Bartlett, 2010; Sewell et al., 2017). 
En el mismo sentido, se ha resaltado que el extensionismo rural es una práctica muy compleja, que suele rebasar una formación profesional específica, dado que los productores trabajan con seres vivos y con alto nivel de riesgo, por lo cual requieren más que el solo asesoramiento productivo, sino que también necesitan de competencias en mercadeo, organización, comunicación así como en temáticas específicas en torno a edafología o salud animal, por citar algunos ejemplos, por lo que es posible que los extensionistas estén llamados a trabajar en equipos y también labrar sus propios esquemas organizativos.

\section{Conclusiones}

En la presente investigación se construyó una tipología de problemas en el desempeño de la labor de los extensionistas en México. A partir de un análisis del perfil de los extensionistas fue posible caracterizar los rasgos generales de la política de extensión en México. Se trata de una política con enfoque sectorial que basa su estrategia en perfiles de los extensionistas en las áreas de agronomía y ganadería. Marginalmente se han empleado extensionistas con perfil de las áreas de biología y ciencias sociales. Además, la evidencia empírica mostró que durante el periodo 2016-2017 el extensionismo en México fue desarrollado principalmente por hombres de mediana edad. A partir del análisis de problemas se identificó un listado de obstáculos que perciben los extensionistas, el cual es un insumo para coadyuvar tanto en un diagnóstico de la política de extensión en México, como de posibles vías para mejorar su eficiencia bajo una visión más amplia.

Adicionalmente, el análisis de clúster agrupó problemas principales por tipo de extensionista según su área profesional, lo que puede auxiliar en el establecimiento de una política de atención a los problemas de los extensionistas de manera diferenciada. Finalmente, esta perspectiva tiene que ser enriquecida con una tipología de los extensionistas más robusta, que agrupe a estos actores según su desempeño a nivel de predio, lo que permitiría caracterizar experiencias exitosas y replicarlas en el marco de la política de extensión en México. Además de la concepción de estos actores, es necesario que se articule con diferentes investigaciones y con evidencia empírica directamente desde los predios de los beneficiarios, con el objetivo de disponer de una valoración multidimensional y tender hacía una visión holística, que es uno de los desafíos del extensionismo rural nacional que se ha delineado en esta investigación.

\section{Literatura citada}

Abdi, H. and Williams, L. 2010. Principal component analysis. Wires computational statistics. 2(4):433-459. Doi:10.1002/wics.101.

Aguilar-Gallegos, N.; Muñoz-Rodríguez, M.; Santoyo-Cortés, H.; Aguilar-Ávila, J. and Klerkx, L. 2015. Information networks that generate economic value: A study on clusters of adopters of new or improved technologies and practices among oil palm growers in Mexico. Agric. Systems. 135(2015):122-132. doi: http://dx.doi.org/10.1016/j.agsy.2015.01.003.

Ardila, J. 2010. Extensión rural para el desarrollo de la agricultura y la seguridad alimentaria: aspectos conceptuales, situación y visión de futuro. Instituto Interamericano de Cooperación para la Agricultura (IIICA). Costa Rica. 128 p.

Bartlett, A. 2010. An introduction to real-world extension. Rural Development News. 1:7-14. 
Bouyer, F.; Hamadou, S.; Adakal, H.; Lancelot, R.; Stachurski, F.; Belem, A. and Bouyer, J. 2011. Restricted application of insecticides: a promising tsetse control technique, but what do the farmers think of it? Plos Negl. Trop. Dis. 5(8):e1276. Doi:10.1371/journal.pntd.0001276.

Christoplos, I. 2010. Cómo movilizar el potencial de la extensión agraria y rural. Organización de las Naciones Unidas para la Agricultura y la Alimentación (FAO). Italia. 68 p.

Cook, B.; Satizábal, P. and Curnow, J. 2021. Humanizing agricultural extension: A Review. World Development. 140:1-19. doi: https://doi.org/10.1016/j.worlddev.2020.105337.

Fantappiè, M.; Lorenzetti, R.; De Meo, I. and Constantini, E. 2020. How to improve the adoption of soil conservation practices? Suggestions from farmers 'perception in western Sicily. J. Rural Studies. 73:186-202. doi: https://doi.org/10.1016/j.jrurstud.2019.11.00.

Greenacre, M. 2007. Correspondence analysis in practice. Chapman \& Hall/CRC. Taylor \& Francis groupo. Second (Ed.). United States of America. 274 p.

Lahai, B.; Goldey, P. and Jones, G. 1999. The gender of the extension agent and farmer'acces to and participation in agricultural extension in nigeria. The J. Agric. Ed. Ext. 6(4):223-233. Doi: 10.1080/13892240085300051.

Lamontagne-Godwin, J.; Williams, F.; Bandara, W. and Appiah-Kubi, Z. 2017. Quality of extension advice: a gender case study from Ghana and Sri Lanka. The J. Agric. Ed. Ext. 23(1):7-22. Doi: 10.1080/1389224X.2016.1230069.

Landini, F. 2016. Problemas de la extensión rural en América Latina. Perfiles Latinoam. 24(47):4768. Doi: 10.18504/pl2447-005-2016.

Qamar, K. 2011. Introducing demand-driven extension approach in a traditional region: a case study fromm Pakistan. Office of knowledge exchange, research and extension. Food and Agriculture Organization of the United Nations (FAO). Rome, Italy. 106 p.

Rogers, E. 2003. Diffusion of innovations. The free press. United States of America. 526 p.

Santos, V.; Álvarez, A. y Francisco, C. 2019. Problemas del extensionismo rural en México. Rev. Geog. Agríc. 62:139-168. doi:dx.doi.org/10.5154/r.rga.2018.62.07.

Santoyo-Cortés, H.; Muñoz-Rodríguez, M.; Aguilar-Ávila, J. y Martínez-González, G. 2016. Extensionismo para la innovación basado en evidencias. In: Martínez-Carrera, D. y Ramírez-Juárez, J. (Ed.). Ciencia tecnología e innovación en el sistema agroalimentario de México. Biblioteca básica de agricultura. Editorial del Colegio de Postgraduados. México, DF. 333-360 pp.

SAGARPA, FAO. 2018. Secretaría de Agricultura, Ganadería, Desarrollo Rural, Pesca y Alimentación y Organización de las Naciones Unidas para la Alimentación y la Agricultura (FAO). 100 p.

SAGARPA, FAO. 2018b. Secretaría de Agricultura, Ganadería, Desarrollo Rural, Pesca y Alimentación y Organización de las Naciones Unidas para la Alimentación y la Agricultura (FAO). 87 p.

Sewell, A.; Hartnett, M.; Gray, D.; Blair, H.; Kemp, P.; Kenyon, P.; Morris, T. and Wood, B. 2017. Using educational theory and research to refine agricultural extension: affordances and barriers for farmers 'learning and practice change. The J. Agric. Ed. Educ. 23(4):313-333. Doi: 10.1080/1389224X.2017.1214861.

Solleiro, J.; Castañón, R. y Martínez, L. 2020. Buenas prácticas de extensionismo y transferencia de tecnología. Recomendaciones para el sistema de extensionismo agroalimentario mexicano. Rev. Mex. Agron. 24(46):508-522.

Urcola, H.; Sartre, X.; Veiga, I.; Elverdin, J. and Albaladejo, C. 2015. Land tenancy, soybean, actors and transformations in the pampas: a district balance. J. Rural Stud. 39(2015):32-40. Doi: http://dx.doi.org/10.1016/j.jrurstud.2015.03.001. 